\title{
WEIERSTRASS EQUATIONS FOR ALL ELLIPTIC FIBRATIONS ON THE MODULAR $K 3$ SURFACE ASSOCIATED TO $\Gamma_{1}(7)$
}

\author{
ODILE LECACHEUX
}

\begin{abstract}
We show that there are 20 elliptic fibrations, up to isomorphism, on the modular $K 3$ surface associated to the modular group $\Gamma_{1}(7)$.
\end{abstract}

1. Introduction. The aim of this paper is to determine all the elliptic fibrations with section up to isomorphism, on the modular surface $S$ associated to $\Gamma_{1}(7)$ and give for each fibration a Weierstrass model.

We prove the following theorem:

Theorem 1.1. There are 20 elliptic fibrations with section up to isomorphism, on the ellipic modular surface associated to the modular group $\Gamma_{1}(7)$.

They are listed in Table 2 with the rank and torsion of their MordellWeil group. The list consists of 1 fibration of rank 0, 18 fibrations of rank 1 and 1 fibration of rank 2 . Among them, five are semi-stable with seven singular fibers, and two of them have the same type of singular fibers but are not isomorphic.

Starting from the elliptic fibration with base curve the modular curve $X_{1}(7)$, we gave, in a previous paper [8], ten elliptic fibrations with elliptic parameters belonging to a multiplicative group.

In this paper, using the lattice-theoretic description of the elliptic fibrations, (Kneser-Nishiyama method) we prove the theorem in the first part (Sections 1-7). Nishiyama's results provide the type of singular fibers, the rank and torsion. In the second part of the paper we

2010 AMS Mathematics subject classification. Primary 11F23, 11G05, 14J28, Secondary 14J27.

Received by the editors on December 17, 2012, and in revised form on September 3, 2013 . 
will give explicit elliptic parameters and Weierstrass equations for the remaining fibrations, using explicit computation of divisors of functions and Elkies's 2 and 3-neighbor method [9, 17].

In [5], Elkies gave a list of negative integers for which there is a unique $K 3$ surface $X$ over $\mathbb{Q}$ with Néron-Severi group of rank 20 and discriminant $-D$ consisting entirely of classes of divisors defined over $\mathbb{Q}$. For $D=-7$, he gave the explicit model of an elliptic fibration

$$
y^{2}=x^{3}-75 x-(64 t+378+64 / t)
$$

with $E_{8}\left(=I I^{*}\right)$ fibers at $t=0$ and $t=\infty$ and an $A_{1}\left(=I_{2}\right)$ fiber at $t=-1$. For this fibration, the Mordell-Weil group has rank 1 and no torsion. We recover Elkies's result, (parameter $h$ from Table 3).

2. Elliptic fibrations. Let $k$ be an algebraically closed field, and let $C$ be a smooth curve over $k$. An elliptic surface $S$ over $C$ is a smooth projective surface $S$ with an elliptic fibration over $C$ means that we have a surjective morphism $f: S \rightarrow C$ such that all but finitely many fibers are smooth curves of genus 1 and no fiber contains an exceptional curve of the first kind (that is, $S \rightarrow C$ is relatively minimal). Moreover, we will assume $f$ has a section, that is, a smooth morphism $s: C \rightarrow S$ such that $f \circ s=\operatorname{id}_{C}$. Let $E$ be the generic fiber; then $E$ can be regarded as an elliptic curve over $K:=k(C)$, the section $s$ corresponding to the $O$ of the elliptic curve. The group $E(K)$ of $K$ rational points of $E$ can be identified with the group of sections of $f$. We also require that $S$ have at least one singular fiber, so, by the Mordell-Weil theorem, $E(K)$ is finitely generated. If $S$ is a $K 3$ surface, then the base curve $C$ is $\mathbb{P}^{1}$ and $K=k(t)$.

The Néron-Severi group $N S(S)$ of $S$, the group of divisors modulo algebraic equivalence, becomes an integral lattice of finite rank $\rho(S)$ with respect to the intersection pairing $(\rho(S)$ is called the Picard number of $S$ ). Let $T$ denote the sublattice (the trivial lattice) of $N S(S)$ generated by the zero section $(O)$ and the irreducible components of fibers. More precisely, the trivial lattice is the orthogonal sum

$$
T=\langle O, F\rangle \oplus_{v \in \sigma} T_{v},
$$

where $O$ denotes the zero section, $F$ the general fiber, $\sigma$ the points of $C$ corresponding to the reducible singular fibers and $T_{v}$ the lattice generated by the fiber components except the zero component. 
The map $P \rightarrow(P) \bmod T$ induces a group isomorphism:

$$
E(K) \simeq N S(S) / T \text {. }
$$

3. Lattices and root lattices. For general definitions concerning lattices, we refer to $[4, \mathbf{1 1}]$.

A lattice is a free abelian group $L$ of finite rank with a non-degenerate symmetric bilinear pairing $b: L \times L \rightarrow \mathbb{Z}$. The lattice is even if $b(x, x) \in 2 \mathbb{Z}$. The pairing $b$ extends to a symmetric bilinear form $\widetilde{b}$ on $L \otimes \mathbb{Q}$. The signature of $b$ is defined to be that of $\widetilde{b}$. For every integer $m$, we denote by $L[m]$ the lattice obtained from a lattice $L$ by multiplying the values of its bilinear form by $m$.

If $S$ is a subset of $L$ we call the orthogonal complement of $S$ the sublattice $S^{\perp}=\{x \in L, b(x, v)=0$, for all $v \in S\}$. A submodule $S \subset L$ is called primitive if the quotient $L / S$ is torsion free. The primitive closure of $S$ in $L$, denoted $\bar{S}^{L}$, is defined by $\{v \in L \mid$ there exists $k \neq$ $0 \in \mathbb{Z}, k v \in S\}$. A homomorphism of lattices $f: S \rightarrow S^{\prime}$ is a homomorphism of abelian groups such that $b^{\prime}(f(x), f(y))=b(x, y)$ for all $x, y \in S$. An injective homomorphism of lattices is called an embedding; an embedding $i$ is called primitive if the quotient $S^{\prime} / i(S)$ is torsion free.

The dual lattice of $L$ is defined by

$$
L^{*}=\operatorname{Hom}(L, \mathbb{Z})=\{x \in L \otimes \mathbb{Q} \mid \forall v \in L, b(v, x) \in \mathbb{Z}\}
$$

and the discriminant group $G_{L}$ is the finite group $L^{*} / L$. If $L^{*}=L$ the lattice is called unimodular. The bilinear form on $L$ induces a symmetric bilinear form called the discriminant bilinear form $b_{L}$ : $G_{L} \times G_{L} \rightarrow \mathbb{Q} / \mathbb{Z}$.

If $L$ is even, then $b_{L}$ is the symmetric bilinear form associated to $q_{L}: G_{L} \rightarrow \mathbb{Q} / 2 \mathbb{Z}$, with $q_{L}(x+L)=b(x, x)+2 \mathbb{Z}$. Then $q_{L}$ is called the discriminant quadratic form.

Let $L$ be a negative-definite lattice (respectively positive-definite), a root $a$ is an element of $L$ satisfying $b(a, a)=-2$ (respectively $b(a, a)=2)$. The sublattice of $L$ generated by the roots is called the root type of $L$ and denoted by $L_{\text {root }}$. If $a$ is a root, the isometry $R_{a}(x)=x+b(x, a) a$ is called the reflection associated to $a$. The subgroup of reflections associated to roots is the Weyl group of $L$. 
Notation 3.1. If $L$ is a lattice, and $x \in L^{*}$, we denote by $\bar{x}$ the element $x+L$ in $L^{*} / L$.

3.1. Lattices $\mathbb{A}_{n}$. If $\left(e_{0}, e_{1}, \ldots, e_{n}\right)$ is the canonical basis of $\mathbb{R}^{n+1}$, we can represent $\mathbb{A}_{n}$ as the lattice

$$
\mathbb{A}_{n}=\left\{\left(x_{0}, x_{1}, \ldots, x_{n}\right) \in \mathbb{Z}^{n+1} \mid \sum_{i=0}^{n} x_{i}=0\right\} .
$$

We take $\left\{\varepsilon_{i}=e_{i}-e_{i-1} \mid i=1,2, \ldots, n\right\}$ as a basis of the lattice $\mathbb{A}_{n}$. The roots of $\mathbb{A}_{n}$ are then the $n(n+1)$ vectors $e_{k}-e_{j} k \neq j$.

Let

$$
\alpha_{1}=\sum_{r=0}^{n-1} \frac{e_{r}}{n+1}-\frac{n e_{n}}{n+1} .
$$

Then

$$
\mathbb{A}_{n}^{*} / \mathbb{A}_{n}=\left\langle\overline{\alpha_{1}}\right\rangle \simeq \mathbb{Z} /(n+1) \mathbb{Z}
$$

3.2. Lattices $\mathbb{D}_{n}$. If $\left(e_{1}, \ldots, e_{n}\right)$ is the canonical basis in $\mathbb{R}^{n}$, we can represent $\mathbb{D}_{n}$ as the lattice

$$
\mathbb{D}_{n}=\left\{\left(x_{1}, x_{2}, \ldots, x_{n}\right) \in \mathbb{Z}^{n+1}, \sum_{i=1}^{n} x_{i} \equiv 0 \bmod 2\right\} .
$$

The lattice $\mathbb{D}_{n}$ is an even lattice. We will use the basis

$$
\delta_{1}=-e_{1}-e_{2}, \delta_{2}=e_{1}-e_{2}, \ldots, \delta_{n}=e_{n-1}-e_{n} .
$$

The roots are the $2 n(n-1)$ vectors $\pm e_{i} \pm e_{k}$.

Let $\widetilde{\delta_{1}}=(1 / 2) \sum_{i=1}^{n} e_{i}$. Then $\widetilde{\delta_{1}}$ is in $\mathbb{D}_{n}^{*}$.

If $n$ is even, then $2 \widetilde{\delta_{1}} \in \mathbb{D}_{n}$; for all $k$, we have $e_{k} \in \mathbb{D}_{n}^{*}$ and $e_{k}-\widetilde{\delta_{1}} \notin \mathbb{D}_{n}$. Then we have

$$
\mathbb{D}_{n}^{*} / \mathbb{D}_{n} \simeq(\mathbb{Z} / 2 \mathbb{Z})^{2}=\left\{0, \overline{\widetilde{\delta_{1}}}, \overline{e_{k}}, \overline{\widetilde{\delta_{1}}+e_{k}}\right\} .
$$

If $n$ is odd, then $2 \widetilde{\delta_{1}} \notin \mathbb{D}_{n}$, and

$$
\mathbb{D}_{n}^{*} / \mathbb{D}_{n} \simeq \mathbb{Z} / 4 \mathbb{Z}=\left\langle\overline{\widetilde{\delta_{1}}}\right\rangle .
$$


3.3. Lattice $\mathbb{E}_{6}$. We can represent $\mathbb{E}_{6}$ as a lattice in $\mathbb{R}^{8}$ generated by the six vectors $\gamma_{i}$

$$
\begin{gathered}
\gamma_{1}=\frac{1}{2}\left(e_{1}+e_{8}\right)-\frac{1}{2} \sum_{i=2}^{7} e_{i}, \quad \gamma_{2}=e_{1}+e_{2}, \gamma_{i}=e_{i-1}-e_{i-2}, \\
3 \leq i \leq 6 .
\end{gathered}
$$

If $\eta_{6}=-(1 / 3)\left(2 \gamma_{1}+3 \gamma_{2}+4 \gamma_{3}+6 \gamma_{4}+5 \gamma_{5}+4 \gamma_{6}\right)$, then

$$
\mathbb{E}_{6}^{*} / \mathbb{E}_{6}=\left\langle\overline{\eta_{6}}\right\rangle \simeq \mathbb{Z} / 3 \mathbb{Z} \text {. }
$$

3.4. Lattice $\mathbb{E}_{7}$. We can represent $\mathbb{E}_{7}$ as a lattice in $\mathbb{R}^{8}$ generated by the six vectors $\gamma_{i}, 1 \leq i \leq 6$, and $\gamma_{7}=e_{6}-e_{5}$.

If $\eta_{7}=(3 / 2)\left(\eta_{6}+\gamma_{7}\right)$, then

$$
\mathbb{E}_{7}^{*} / \mathbb{E}_{7}=\left\langle\overline{\eta_{7}}\right\rangle \simeq \mathbb{Z} / 2 \mathbb{Z}
$$

3.5. Lattice $\mathbb{E}_{8}$. The lattice $\mathbb{E}_{8}$ is the unimodular lattice represented in $\mathbb{R}^{8}$ by points with coordinates $x_{i}$ satisfying

$$
\sum_{i=1}^{8} x_{i} \in 2 \mathbb{Z}, \quad x_{i}-x_{j} \in \mathbb{Z}, 2 x_{i} \in \mathbb{Z} .
$$

4. Nishiyama and Nikulin's results. Nishiyama [14] has classified all possible Jacobian fibrations (fibrations with a section) on some singular $K 3$ surface, i.e., $K 3$ surface of Picard number 20. We summarize the main points of Nishiyama's construction.

Denote by $\mathbb{T}(S)$ the transcendental lattice of $S$, i.e., the orthogonal complement of $N S(S)$ in the unimodular lattice $H^{2}(S, \mathbb{Z})$ with respect to the cup-product,

$$
\mathbb{T}(S)=N S(S)^{\perp} \subset H^{2}(S, \mathbb{Z}) .
$$

Since $\rho(S)=20, \mathbb{T}(S)$ is an even lattice of rank $22-\rho(S)=2$ and signature $(2,0)$ and $\mathbb{T}(S)[-1]$ is an even negative-definite lattice.

By Nikulin's result ([13, Theorem 1.12.4]), $\mathbb{T}(S)[-1]$ admits a primitive embedding into the unimodular lattice $\mathbb{E}_{8}[-1]$.

We define $\underline{M}$ as the orthogonal complement of $\mathbb{T}(S)[-1]$ in $\mathbb{E}_{8}[-1]$. By construction, $\underline{M}$ is a negative-definite lattice of rank 6 . By Nikulin's 
result ([13, Proposition 1.5.1]) the discriminant forms satisfy

$$
q_{\mathbb{T}(S)}=-q_{N S(S)}, \quad q_{\underline{M}}=-q_{\mathbb{T}(S)[-1]} .
$$

For each elliptic fibration $f: S \rightarrow \mathbb{P}^{1}$ of $S$ with a section $O$, we define an even unimodular lattice of signature $(1,1)$ generated by the zero section $O$ and the general fiber $F$, called the hyperbolic lattice $U$. The orthogonal complement lattice of $U$ in the Néron-Severi group $N S(S)$ is called the frame and is denoted $W(S)$. Since $U$ is unimodular, we have $N S(S)=U \oplus W(S)$. The frame is a negative definite even lattice of rank $\rho(S)-2=18$. From Nishiyama's result [14, Lemma $6.3]$, the discriminant groups $G_{\mathbb{T}(S)}$ and $G_{W(S)}$ are isomorphic and $q_{W(S)}=-q_{\mathbb{T}(S)}$.

From Nikulin's result ([13, Corollary 1.6.2]) there exists a unimodular overlattice $L$ of $\underline{M} \oplus W(S)$ such that the embeddings of $\underline{M}$ and $W(S)$ in $L$ are primitive, and $\underline{M}_{L}^{\perp}=W(S)$ and $W(S)_{L}^{\perp}=\underline{M}$. In our case, $L$ is a unimodular lattice of rank 24 . Such a lattice is called a Niemeier lattice, and there are only 24 different Niemeier lattices up to isometry ([12]). A Niemeier lattice $L$ is determined by its root type $L_{\text {root }}$. They are listed in Table 4.

Conversely, if there is a primitive embedding of $\underline{M}$ in a Niemeier lattice, there is an elliptic fibration whose properties will be specified in the following paragraphs.

From now on, we are interested in the $K 3$ elliptic modular surface with base curve the modular curve $X_{1}(7)$ (for equations, see (9.1) below).

The Gram matrix of $\mathbb{T}(S)$ is ([6, Table 1])

$$
\left(\begin{array}{ll}
2 & 1 \\
1 & 4
\end{array}\right) \text {. }
$$

From Nishiyama's computations [14, page 311], we get

$$
\underline{M}[-1]=\mathbb{A}_{6} .
$$

So $\underline{M}=\underline{M}_{\text {root }}$, and we can apply Nishiyama's result: $\underline{M}$ is primitively embedded in $L$ if and only if $\underline{M}$ is primitively embedded in $L_{\text {root }}[\mathbf{1 4}$, page 344]. 


\section{Primitive embeddings of $\mathbb{A}_{6}$.}

Lemma 5.1. Up to the action of the Weyl group, there is at most one primitive embedding $i$ of $\mathbb{A}_{6}$ into $\mathbb{A}_{n}, \mathbb{D}_{m}, \mathbb{E}_{k}$.

(i) The primitive embeddings $i\left(\mathbb{A}_{6}\right)$ of $\mathbb{A}_{6}$ into $\mathbb{A}_{n}$ and $n \geq 6$, up to the action of group $W\left(\mathbb{A}_{n}\right)$, are uniquely given as

$$
i\left(\mathbb{A}_{6}\right)=\left\langle\varepsilon_{1}, \varepsilon_{2}, \ldots, \varepsilon_{6}\right\rangle .
$$

Moreover, the root lattice of $i\left(\mathbb{A}_{6}\right)^{\perp}$ in $\mathbb{A}_{n}$ is isometric to $\mathbb{A}_{n-7}$, for $n>7$ and 0 if $n \leq 7$.

(ii) The primitive embeddings $i\left(\mathbb{A}_{6}\right)$ of $\mathbb{A}_{6}$ into $\mathbb{D}_{m}$ and $m \geq 7$, up to the action of group $W\left(\mathbb{D}_{m}\right)$, is uniquely given as

$$
i\left(\mathbb{A}_{6}\right)=\left\langle\delta_{2}, \delta_{3}, \ldots, \delta_{7}\right\rangle .
$$

Moreover, the root lattice of $i\left(\mathbb{A}_{6}\right)^{\perp}$ in $\mathbb{D}_{m}$ is isometric to $\mathbb{D}_{m-7}$, for $m>7$ and 0 if $m \leq 7$.

(iii) The primitive embeddings $i\left(\mathbb{A}_{6}\right)$ of $\mathbb{A}_{6}$ into $\mathbb{E}_{k}=\left\langle\eta_{i}, i=1, \ldots, k\right\rangle$ and $k \geq 7$, up to the action of group $W\left(\mathbb{E}_{k}\right)$, is uniquely given as

$$
i\left(\mathbb{A}_{6}\right)=\left\langle\eta_{2}, \eta_{3}, \ldots, \eta_{7}\right\rangle
$$

TABLE 1. Niemeier lattices.

\begin{tabular}{|l|l|l|l|l|l|}
\hline Name & $L_{\text {roots }}$ & $L / L_{\text {roots }}$ & Name & $L_{\text {roots }}$ & $L / L_{\text {roots }}$ \\
\hline$\alpha$ & $\mathbb{D}_{24}$ & $\mathbb{Z} / 2 \mathbb{Z}$ & $\nu$ & $\mathbb{A}_{9}^{2} \oplus \mathbb{D}_{6}$ & $\mathbb{Z} / 2 \mathbb{Z} \times \mathbb{Z} / 10 \mathbb{Z}$ \\
\hline$\beta$ & $\mathbb{D}_{16} \oplus \mathbb{E}_{8}$ & $\mathbb{Z} / 2 \mathbb{Z}$ & $\xi$ & $\mathbb{D}_{6}^{4}$ & $(\mathbb{Z} / 2 \mathbb{Z})^{4}$ \\
\hline$\gamma$ & $\mathbb{E}_{8}^{3}$ & 0 & $o$ & $\mathbb{A}_{8}^{3}$ & $(\mathbb{Z} / 3 \mathbb{Z})^{3}$ \\
\hline$\delta$ & $\mathbb{A}_{24}$ & $\mathbb{Z} / 5 \mathbb{Z}$ & $\pi$ & $\mathbb{A}_{7}^{2} \oplus \mathbb{D}_{5}^{2}$ & $\mathbb{Z} / 4 \mathbb{Z} \times \mathbb{Z} / 8 \mathbb{Z}$ \\
\hline$\varepsilon$ & $\mathbb{D}_{12}^{2}$ & $(\mathbb{Z} / 2 \mathbb{Z})^{2}$ & $\rho$ & $\mathbb{A}_{6}^{4}$ & $(\mathbb{Z} / 7 \mathbb{Z})^{2}$ \\
\hline$\zeta$ & $\mathbb{A}_{17} \oplus \mathbb{E}_{7}$ & $\mathbb{Z} / 6 \mathbb{Z}$ & $\sigma$ & $\mathbb{A}_{5}^{4} \oplus \mathbb{D}_{4}$ & $\mathbb{Z} / 2 \mathbb{Z} \times(\mathbb{Z} / 6 \mathbb{Z})^{2}$ \\
\hline$\eta$ & $\mathbb{D}_{10} \oplus \mathbb{E}_{7}^{2}$ & $(\mathbb{Z} / 2 \mathbb{Z})^{2}$ & $\tau$ & $\mathbb{D}_{4}^{6}$ & $(\mathbb{Z} / 2 \mathbb{Z})^{6}$ \\
\hline$\theta$ & $\mathbb{A}_{15} \oplus \mathbb{D}_{9}$ & $\mathbb{Z} / 8 \mathbb{Z}$ & $v$ & $\mathbb{A}_{4}^{6}$ & $(\mathbb{Z} / 5 \mathbb{Z})^{3}$ \\
\hline$\iota$ & $\mathbb{D}_{8}^{3}$ & $(\mathbb{Z} / 2 \mathbb{Z})^{3}$ & $\phi$ & $\mathbb{A}_{3}^{6}$ & $(\mathbb{Z} / 4 \mathbb{Z})^{4}$ \\
\hline$\kappa$ & $\mathbb{A}_{12}^{2}$ & $\mathbb{Z} / 13 \mathbb{Z}$ & $\chi$ & $\mathbb{A}_{2}^{12}$ & $(\mathbb{Z} / 3 \mathbb{Z})^{6}$ \\
\hline$\lambda$ & $\mathbb{A}_{11} \oplus \mathbb{D}_{7} \oplus \mathbb{E}_{6}$ & $\mathbb{Z} / 12 \mathbb{Z}$ & $\psi$ & $\mathbb{A}_{1}^{24}$ & $(\mathbb{Z} / 2 \mathbb{Z})^{12}$ \\
\hline$\mu$ & $\mathbb{E}_{6}^{4}$ & $(\mathbb{Z} / 3 \mathbb{Z})^{2}$ & $\omega$ & Leech & $\Lambda_{24}$ \\
\hline
\end{tabular}


Moreover, the root lattice of $i\left(\mathbb{A}_{6}\right)^{\perp}$ in $\mathbb{E}_{k}$ is 0 for $k=7$, isometric to $\mathbb{A}_{1}$ for $k=8$.

In other cases, $\mathbb{A}_{6}$ cannot be embedded.

Proof. We use Nishiyama's results [14, Lemmas 4.1, 4.2 and 4.3] to give the embeddings. Then, using the roots of each of lattices $\mathbb{A}_{n}$ and $\mathbb{D}_{m}$, we can compute which roots are in $i\left(\mathbb{A}_{6}\right)^{\perp}$. For $\mathbb{E}_{k}$, see Nishiyama [14, Corollary 4.4].

6. All the fibrations. All the fibrations are obtained in embedding $\mathbb{A}_{6}$ in one factor of $L_{\text {root }}$. The results are gathered in the Table 2. In each case $\mathbb{A}_{6}$ is embedded in the factor of $L_{\text {root }}$ listed first. This first factors are successively $\mathbb{A}_{n}, \mathbb{D}_{m}$ and $\mathbb{E}_{k}$.

The following results [14, Lemma 6.1 and definitions] allow us to obtain some properties of the fibration derived from a primitive embedding of $\mathbb{A}_{6}$ in $L$.

\section{Lemma 6.1.}

(i) The type $\oplus_{v \in \sigma} T_{v}$ of a fibration is isomorphic to the type $W(S)_{\text {root }}$.

(ii) The Mordell-Weil group of a fibration is isomorphic to

$$
\frac{W(S)}{W(S)_{\text {root }}} \text {. }
$$

(iii) The torsion in the Mordell-Weil group is isomorphic to

$$
\frac{\overline{\left(W(S)_{\text {root }}\right)}}{W(S)_{\text {root }}}
$$

where $\overline{\left(W(S)_{\text {root }}\right)}$ is the primitive closure of $W(S)_{\text {root }}$ in $W(S)$.

In all cases of Table 2, the rank of the Mordell-Weil group is $>0$ except for $L_{\text {root }}=\mathbb{A}_{6}^{4}$. Moreover, the rank is one except for $L_{\text {root }}=\mathbb{D}_{8}^{3}$ (parameter $t$ ) for which the rank is 2 . Finally the second column of Table 2 returns to equations of Tables 3 and 4 . 
TABLE 2. All the elliptic fibrations.

\begin{tabular}{|c|c|c|c|}
\hline First case of Lemma 1 & parameter & Singular fibers & Torsion \\
\hline$\rho: \mathbb{A}_{6}^{4}$ & $d$ & $3 I_{7}, 3 I_{1}$ & $(\mathbb{Z} / 7 \mathbb{Z})$ \\
\hline$\pi: \mathbb{A}_{7}^{2} \oplus \mathbb{D}_{5}^{2}$ & $u$ & $I_{8}, 2 I_{1}^{*}, 2 I_{1}$ & $(\mathbb{Z} / 4 \mathbb{Z})$ \\
\hline$o: \mathbb{A}_{8}^{3}$ & $r$ & $I_{2}, 2 I_{9}, 4 I_{1}$ & $(\mathbb{Z} / 3 \mathbb{Z})$ \\
\hline$\nu: \mathbb{A}_{9}^{2} \oplus \mathbb{D}_{6}$ & $k$ & $I_{3}, I_{10}, I_{2}^{*}, 3 I_{1}$ & $(\mathbb{Z} / 2 \mathbb{Z})$ \\
\hline$\lambda: \mathbb{A}_{11} \oplus \mathbb{D}_{7} \oplus \mathbb{E}_{6}$ & $s$ & $I_{5}, I_{3}^{*}, I V^{*}, 2 I_{1}$ & 0 \\
\hline$\chi: \mathbb{A}_{12}^{2}$ & $w$ & $I_{6}, I_{13}, 5 I_{1}$ & 0 \\
\hline$\theta: \mathbb{A}_{15} \oplus \mathbb{D}_{9}$ & $p$ & $I_{9}, I_{5}^{*}, 4 I_{1}$ & 0 \\
\hline$\zeta: \mathbb{A}_{17} \oplus \mathbb{E}_{7}$ & $a$ & $I_{11}, I I I^{*}, 4 I_{1}$ & 0 \\
\hline $\mathbb{A}_{24}$ & $g$ & $I_{18}, 6 I_{1}$ & 0 \\
\hline Second case & & & \\
\hline$\lambda: \mathbb{D}_{7} \oplus \mathbb{A}_{11} \oplus \mathbb{E}_{6}$ & $m$ & $I_{12}, I V^{*}, 4 I_{1}$ & $(\mathbb{Z} / 3 \mathbb{Z})$ \\
\hline$\iota: \mathbb{D}_{8}^{3}$ & $t$ & $2 I_{4}^{*}, 4 I_{1}$ & $(\mathbb{Z} / 2 \mathbb{Z})$ \\
\hline$\theta: \mathbb{D}_{9} \oplus \mathbb{A}_{15}$ & $b$ & $2 I_{2}, I_{16}, 4 I_{1}$ & $(\mathbb{Z} / 4 \mathbb{Z})$ \\
\hline$\eta: \mathbb{D}_{10} \oplus \mathbb{E}_{7}^{2}$ & $n$ & $I_{4}, 2 I I I^{*}, 2 I_{1}$ & $(\mathbb{Z} / 2 \mathbb{Z})$ \\
\hline$\epsilon: \mathbb{D}_{12}^{2}$ & $q$ & $I_{1}^{*}, I_{8}^{*}, 3 I_{1}$ & $(\mathbb{Z} / 2 \mathbb{Z})$ \\
\hline$\beta: \mathbb{D}_{16} \oplus \mathbb{E}_{8}$ & $l$ & $I_{5}^{*}, I I^{*}, 3 I_{1}$ & 0 \\
\hline $\mathbb{D}_{24}$ & $e$ & $I_{13}^{*}, 5 I_{1}$ & 0 \\
\hline Third Case & & & \\
\hline$\zeta: \mathbb{E}_{7} \oplus \mathbb{A}_{17}$ & $f$ & $I_{18}, 6 I_{1}$ & $(\mathbb{Z} / 3 \mathbb{Z})$ \\
\hline$\eta: \mathbb{E}_{7}^{2} \oplus \mathbb{D}_{10}$ & $c$ & $I I I^{*}, I_{6}^{*}, 3 I_{1}$ & $(\mathbb{Z} / 2 \mathbb{Z})$ \\
\hline$\beta: \mathbb{E}_{8} \oplus \mathbb{D}_{16}$ & $o$ & $I_{2}, I_{12}^{*}, 4 I_{1}$ & $(\mathbb{Z} / 2 \mathbb{Z})$ \\
\hline$\gamma: \mathbb{E}_{8}^{3}$ & $h$ & $I_{2}, 2 I I^{*}, 2 I_{1}$ & 0 \\
\hline
\end{tabular}

\section{Torsion.}

7.1. Methods. Let $\underline{M}$ be a primitive embedding of $\mathbb{A}_{6}$ in $L_{\text {root }}, M$ the orthogonal complement of $\underline{M}$ in $L_{\text {root }}$, and let $W$ be the orthogonal complement of $\underline{M}$ in $L$.

From elementary algebra, we have the following properties

(i) $M=L_{\text {root }} \cap W$ so $W / M=W / L_{\text {root }} \cap W$,

(ii) from the canonical surjection $L \rightarrow L / L_{\text {root }}$ we get the injection

$$
W / M \hookrightarrow L / L_{\text {root }},
$$


(iii) since $L / L_{\text {root }}$ is finite, $W / M$ is also finite of order $i_{W, M}$ and

$$
i_{W, N}^{2} \operatorname{det} W=\operatorname{det} M,
$$

(iv) from the canonical surjection $W \rightarrow W / M$ we have the injection

$$
\overline{W_{\text {root }}} / \overline{W_{\text {root }}} \cap M \hookrightarrow W / M .
$$

From definitions we have $M_{\text {root }}=W_{\text {root }}$, and from Nishiyama's results

(a) $\operatorname{det} W=\operatorname{det} M=7$,

(b) $M / M_{\text {root }}$ is torsion free, so ${\overline{M_{\text {root }}}}^{M}=M_{\text {root }}$.

Finally, we have the equality $\overline{W_{\text {root }}} \cap M=\overline{M_{\text {root }}} \cap M={\overline{M_{\text {root }}}}^{M}$. So, we have an injection

$$
\overline{W_{\text {root }}} / W_{\text {root }} \hookrightarrow W / M .
$$

7.2. Computations. Let $L_{\text {root }}=A \oplus B$ with a primitive embedding of $\mathbb{A}_{6}$ in an indecomposable root lattice $A$, and let $B$ be a direct sum of the root lattices. If $M_{1}$ is the orthogonal complement of $\mathbb{A}_{6}$ in $A$, then $M=M_{1} \oplus B$ and $M_{\text {root }}=\left(M_{1}\right)_{\text {root }} \oplus B$.

Since $W / M$ is a finite group, we have $r:=\operatorname{rank} W-\operatorname{rank} W_{\text {root }}=$ $\operatorname{rank} M_{1}-\operatorname{rank}\left(M_{1}\right)_{\text {root }}$. We use the generators of $L / L_{\text {root }}$ given in [4] as elements of $L_{\text {root }}^{*} / L_{\text {root }}$.

7.3. $A=\mathbb{A}_{n}$. If $n \geq 7$, we have $\operatorname{rank}\left(M_{1}\right)-\operatorname{rank}\left(M_{1}\right)_{\text {root }}=(n-6)-$ $(n-7)=1$ and $W_{\text {root }}=\mathbb{A}_{n-7} \oplus B$.

Since $\bar{W}_{\text {root }}$ is an overlattice of $W_{\text {root }}$, it can be viewed as a subgroup of $W_{\text {root }}^{*} / W_{\text {root }}=\mathbb{A}_{n-7}^{*} / \mathbb{A}_{n-7} \oplus B^{*} / B$. Moreover, using the injection

$$
\overline{W_{\text {root }}} / W_{\text {root }} \hookrightarrow L_{\text {root }}^{*} / L_{\text {root }} \hookrightarrow \mathbb{A}_{n}^{*} / \mathbb{A}_{n} \oplus B^{*} / B,
$$

we see that the first component of $u \in \overline{W_{\text {root }}} / W_{\text {root }}$ is of order dividing $n-6$ and $n+1$, so dividing 7 . In all cases, treated $n+1$ is never divided by 7 , so we have an injection

$$
\overline{W_{\text {root }}} / W_{\text {root }} \hookrightarrow B^{*} / B .
$$

Conversely, if $x \in L / L_{\text {root }}$, represented by $(u, v)$ with $u \in A^{*} / A, v \in$ $B^{*} / B$, then $(u, v) \in \overline{W_{\text {root }}} / W_{\text {root }}$ if and only if $u=0$. 
(i) If $n=6$, then $L_{\text {root }}=\mathbb{A}_{6}^{4}$; thus, the rank is 0 and $\operatorname{det} M=7^{3}$. Since $\operatorname{det} W=7$ then $|W / M|^{2}=\operatorname{det} M / \operatorname{det} W=7^{2}$. Then the torsion group is cyclic of order 7 .

(ii) If $n=7$ and $L_{\text {root }}=\mathbb{A}_{7}^{2} \oplus \mathbb{D}_{5}^{2}$, then the group $L / L_{\text {root }}$ is of order 32 isomorphic to $\mathbb{Z} / 8 \mathbb{Z} \times \mathbb{Z} / 4 \mathbb{Z}$, with two generators chosen as $\left(\overline{\alpha_{1}}, \overline{\alpha_{1}}, \overline{\delta_{1}}, 2 \overline{\delta_{1}}\right)$ and $\left(2 \overline{\alpha_{1}}, 4 \overline{\alpha_{1}}, \overline{\delta_{1}}, \overline{\delta_{1}}\right)$. Enumerating all the elements, we see that only 4 elements have $\overline{0}$ as the first component: $0, x=\left(\overline{0}, 4 \overline{\alpha_{1}}, 2 \overline{\delta_{1}}, 2 \overline{\delta_{1}}\right), y=\left(\overline{0}, 6 \overline{\alpha_{1}}, \overline{\delta_{1}}, 3 \overline{\delta_{1}}\right),-y$. Since $2 y=x$, the torsion group is cyclic of order 4 .

(iii) If $n=8$ and $L_{\text {root }}=\mathbb{A}_{8}^{3}$, then the group $L / L_{\text {root }}$ is isomorphic to $\mathbb{Z} / 9 \mathbb{Z} \times \mathbb{Z} / 3 \mathbb{Z}$ generated by the three elements: $\left(\overline{\alpha_{1}}, \overline{\alpha_{1}}, 4 \overline{\alpha_{1}}\right)$, $\left(\overline{\alpha_{1}}, 4 \overline{\alpha_{1}}, \overline{\alpha_{1}}\right)$, and $\left(4 \overline{\alpha_{1}}, \overline{\alpha_{1}}, \overline{\alpha_{1}}\right)$. Enumerating all the elements, we see that only three elements have the first component equal to $\overline{0}$. So the torsion group is cyclic of order 3 .

(iv) If $n=9$ and $L_{\text {root }}=\mathbb{A}_{9}^{2} \oplus \mathbb{D}_{6}$, then the group $L / L_{\text {root }}$ is of order 20 generated by $\left(2 \overline{\alpha_{1}}, 4 \overline{\alpha_{1}}, 0\right),\left(5 \overline{\alpha_{1}}, 0, \overline{\widetilde{\delta_{1}}}\right),\left(0,5 \overline{\alpha_{1}}, \overline{\widetilde{\delta_{1}}+e_{2}}\right)$. Only two elements have a first component equal to 0 so the torsion group is cyclic of order 2 .

(v) For $n=11,12,15,17,24$, only one element has a first component equal to $\overline{0}$, so the torsion group is trivial.

7.4. $A=\mathbb{D}_{m}$. For $m>9$, we have $\left(M_{1}\right)_{\text {root }}=\mathbb{D}_{m-7}$, and we can see $\overline{\left(M_{1}\right)_{\text {root }}} /\left(M_{1}\right)_{\text {root }}$ as a subgroup of $\mathbb{D}_{m-7}^{*} / \mathbb{D}_{m-7}$. Since $m$ and $m-7$ do not have the same parity, the first component of elements of $\overline{W_{\text {root }}} / W_{\text {root }}$ in $L / L_{\text {root }}$ is in a fixed group of order 2 .

(i) If $m=7$ and $L_{\text {root }}=\mathbb{D}_{7} \oplus \mathbb{A}_{11} \oplus \mathbb{E}_{6}$, we have $\left(M_{1}\right)_{\text {root }}=0$, and so the rank is 1 and the first component of elements of $\overline{W_{\text {root }}} / W_{\text {root }}$ in $L / L_{\text {root }}$ is 0 . Moreover, $L / L_{\text {root }}$ is cyclic of order 12 generated by $\left(\widetilde{\widetilde{\delta}}_{1}, \overline{\alpha_{1}}, \overline{\eta_{6}}\right)$. Since $\overline{\widetilde{\delta}_{1}}$ is of order 4 , there are three elements with first component equal to 0 . Thus, the torsion group is equal to $\mathbb{Z} / 3 \mathbb{Z}$.

(ii) If $m=8$ and $L_{\text {root }}=\mathbb{D}_{8}^{3}$, the lattice $M_{1}$ is of rank 2 and $\left(M_{1}\right)_{\text {root }}=0$, so the rank is 2 . As for $m=7$, we have to consider elements with first component equal to 0 . The group $L / L_{\text {root }} \simeq(\mathbb{Z} / 2 \mathbb{Z})^{3}$ is of order 8 generated by $r_{1}=\left(\overline{\widetilde{\delta_{1}}}, \overline{\delta_{2}, \delta_{2}}\right)$, $r_{2}=\left(\overline{\delta_{2}}, \overline{\widetilde{\delta_{1}}} \overline{\delta_{2}}\right)$ and $r_{3}=\left(\overline{\delta_{2}}, \overline{\delta_{2}}, \overline{\widetilde{\delta_{1}}}\right)$ with $\widetilde{\delta_{1}}=(1 / 2) \sum_{i=1}^{8} e_{i}$ and 
$\delta_{2}=e_{2}$. Only $r_{2}+r_{3}$ has the desired property, so the torsion group is equal to $\mathbb{Z} / 2 \mathbb{Z}$.

(iii) For $m=9$ and $L_{\text {root }}=\mathbb{D}_{9} \oplus \mathbb{A}_{15}$, we have $\left(M_{1}\right)_{\text {root }}=\mathbb{A}_{1}^{2}$, so the rank is 1 . The first factor of $\overline{W_{\text {root }}} / W_{\text {root }}$ is a subgroup of $\mathbb{D}_{9}^{*} / \mathbb{D}_{9}$; this last group is cyclic of order 4 . It can also be viewed as a subgroup of the order 4 cyclic group $\mathbb{D}_{9}^{*} / \mathbb{D}_{9}$. Since $L / L_{\text {root }}$ is cyclic of order 8 generated by $\left(\overline{\delta_{1}}, 2 \overline{\alpha_{1}}\right)$ with $\overline{\delta_{1}}$ of order 4 , $\overline{W_{\text {root }}} / W_{\text {root }}$ is cyclic of order 4 generated by $2\left(\overline{\delta_{1}}, 2 \overline{\alpha_{1}}\right)$.

(iv) For the remaining cases $m=10,12,16,24$, we have to consider the quotient $\mathbb{D}_{m}^{*} / \mathbb{D}_{m}=\left\{0, \overline{\widetilde{\delta_{1}}}, \overline{e_{i}}, \overline{\widetilde{\delta_{1}}+e_{i}}\right\}$. We know that $2 \overline{e_{i}} \in \mathbb{D}_{m}$. Moreover, if we consider the primitive embedding of $\mathbb{A}_{6}$ in $\mathbb{D}_{m}$, $i\left(\mathbb{A}_{6}\right)=\left\langle\delta_{2}, \delta_{3}, \ldots, \delta_{7}\right\rangle$ with $\delta_{i+1}=e_{i}-e_{i+1}$, then $\left(M_{1}\right)_{\text {root }}=$ $\left\langle e_{8}+e_{9}, \delta_{9}, \ldots, \delta_{m}\right\rangle$. If we choose $i=8$, we can see that $2 \overline{e_{8}} \in \mathbb{D}_{m}$ and, for all $k, k \widetilde{\delta_{1}} \notin\left(M_{1}\right)_{\text {root }}$ and $k\left(\widetilde{\delta_{1}}+e_{i}\right) \notin\left(M_{1}\right)_{\text {root }}$. So, for these four cases, an element of $L / L_{\text {root }}$ belongs to $\overline{W_{\text {root }}} / W_{\text {root }}$ if and only if the first component is 0 or $\overline{e_{i}}$. After examination of each case $m=10,12,16,24$ we can complete the table.

7.5. $A=\mathbb{E}_{k}$.

(i) $L_{\text {root }}=\mathbb{E}_{7} \oplus \mathbb{A}_{17}$. In that case $L / L_{\text {root }}$ is cyclic of order 6 generated by $\left(\overline{\eta_{7}}, 3 \overline{\alpha_{1}}\right)$. Since $\left(M_{1}\right)_{\text {root }}=0$, the elements of $\overline{W_{\text {root }}} / W_{\text {root }}$ have their first component equal to 0 , so $\overline{W_{\text {root }}} / W_{\text {root }}$ is of order 2 .

(ii) $L_{\text {root }}=\mathbb{E}_{7}^{2} \oplus \mathbb{D}_{10}$. The group $L / L_{\text {root }}$ previously described is, after permutation, isomorphic to $(\mathbb{Z} / 2 \mathbb{Z})^{2}$. The non zero elements are $\left(\overline{\eta_{7}}, 0, u\right),\left(0, \overline{\eta_{7}}, u^{\prime}\right)$ and $\left(\overline{\eta_{7}}, \overline{\eta_{7}}, u^{\prime \prime}\right)$. Since $\left(M_{1}\right)_{\text {root }}=0$, only $\left(0, \overline{\eta_{7}}, u^{\prime}\right)$ belongs to $\overline{W_{\text {root }}} / W_{\text {root }}$, so the torsion group is of order 2 .

(iii) $L_{\text {root }}=\mathbb{E}_{8} \oplus \mathbb{D}_{16}$. The group $L / L_{\text {root }}$ is isomorphic to $\mathbb{Z} / 2 \mathbb{Z}$, generated by $v=\left(0, \overline{\widetilde{\delta_{1}}}\right)$ and $2 v \in W_{\text {root }}$, so the torsion group is of order 2 .

(iv) $L_{\text {root }}=\mathbb{E}_{8}^{3}$. Then $L / L_{\text {root }}=0$, so the torsion group is 0 .

8. Computations of Weierstrass models. We will use the following proposition ([15, pages 559-560] or [16, Proposition 12.10]).

Proposition 8.1. Let $S$ be a $K 3$ surface and $D$ an effective divisor 
on $S$ that has the same type as a singular fiber of an elliptic fibration. Then $S$ admits a unique elliptic fibration with $D$ as a singular fiber. Moreover, any irreducible curve $C$ on $S$ with D.C $=1$ induces a section of the elliptic fibration.

If $S$ is a $K 3$ surface, an elliptic fibration $f: S \rightarrow \mathbb{P}^{1}$ with a section 0 defines a non constant function $t$, with $t=f(z)$ for $z \in S$; the function $t$ is called the elliptic parameter. Then the generic fiber $E$ has a Weierstrass equation on $k(t)$. The parameter $t$ is unique only up to linear fractional transformations. From Proposition 1, to construct a fibration, we need one effective divisor $D$. We call such a divisor an elliptic divisor. In practice, however, we need two divisors $D_{1}$ and $D_{2}$, one for $t=0$ and the other for $t=\infty$, as in the next proposition.

Proposition 8.2. Let $S$ be a $K 3$ surface and $f: X \rightarrow \mathbb{P}^{1}$ an elliptic fibration of elliptic parameter $t$. Let $\Delta$ be a set of components of singular fibers included in $f^{-1}(W)$ where $W$ is a finite subset of $\mathbb{P}^{1}$ and $\delta$ a finite set of sections. Suppose $D_{1}$ and $D_{2}$ are two elliptic divisors of the same new fibration with supports contained in $\Delta \cup \delta$. If we write $D_{i}=\delta_{i}+\Delta_{i}$ with $\delta_{i}$ the sum of sections and $\Delta_{i}$ the sum of components of singular fibers, then $\delta_{1}-\delta_{2}$ is the divisor of a function $u_{0}$ on the generic fiber $E(k(t))$ and a parameter of the new fibration can be chosen as the function $u=u_{0} \prod_{t_{i} \in W}\left(t-t_{i}\right)^{a_{i}}$ where $a_{i} \in \mathbb{Z}$.

Proof. In the new fibration, there is a function $u$ with divisor $D_{1}-D_{2}$ so $D_{1}$ and $D_{2}$ belong to the same class in $N S(S)$. As the class of $\Delta_{1}-\Delta_{2}$ is in $T$, then $\delta_{1}$ and $\delta_{2}$ are in the same class in $N S(S) / T$. Using the isomorphism between $N S(S) / T$ and $E(k(t))$, it follows that $\delta_{1}-\delta_{2}$ is the divisor of a function $u_{0}$ on $E(k(t))$.

\section{Equations.}

9.1. Previous results. We recall some notation and results from [8]. We start with the elliptic modular surface

$$
\mathcal{Y}^{2}+\left(1+d-d^{2}\right) \mathcal{X} \mathcal{Y}+\left(d^{2}-d^{3}\right) \mathcal{Y}=\mathcal{X}^{3}+\left(d^{2}-d^{3}\right) \mathcal{X}^{2}
$$

and the elliptic fibration

$$
(\mathcal{X}, \mathcal{Y}, d) \longmapsto d
$$


where $A=(0,0)$ is a 7 -torsion point. Moreover, at $d=1,0, \infty$, we have singular fibers of type $I_{7}$. The components of singular fibers of type $I_{7}$ at $d \in 0,1, \infty$ are denoted by $\Theta_{d, j}, j=0,1, \ldots, 6$, and the Coxeter graph of sections and $\Theta_{d, j}$ components can be drawn as below.

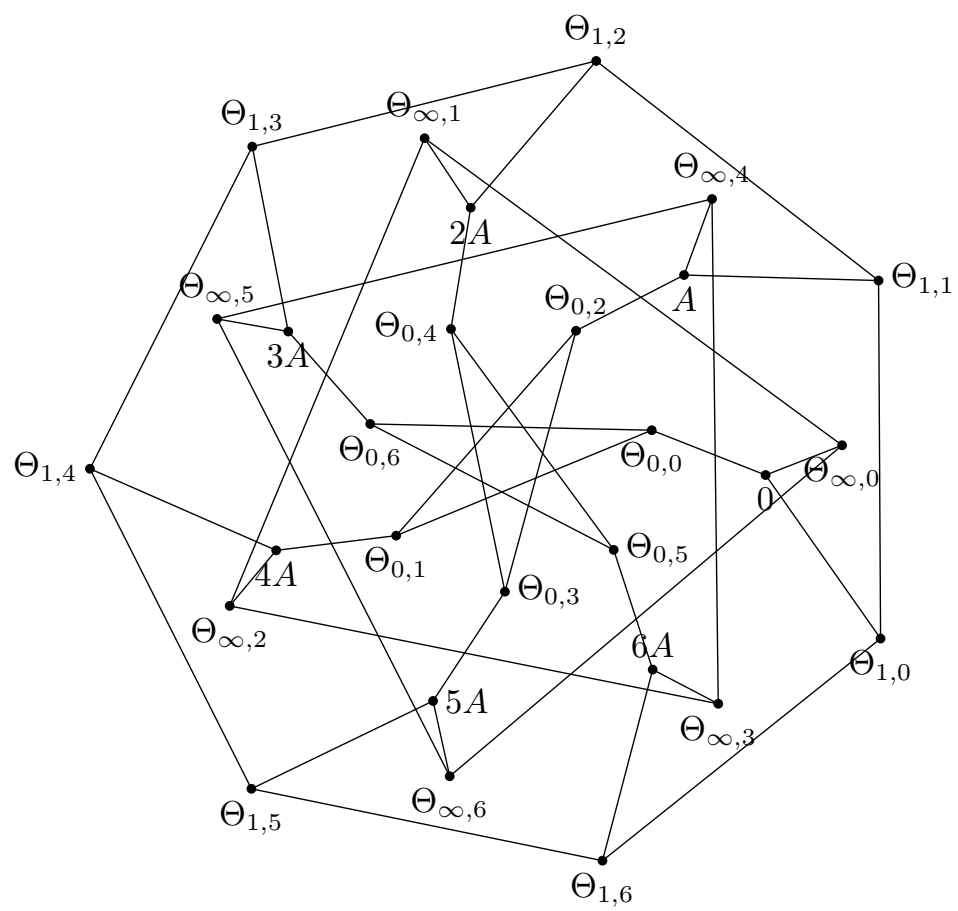

Using the birational transformation

$$
u=\frac{\mathcal{Y}}{\mathcal{X}^{2}}, \quad v=-\frac{\mathcal{X}+d^{2}-d^{3}}{\mathcal{Y}}
$$

with inverse

$$
\mathcal{X}=\frac{d(d-1)}{u+v-u v}, \quad \mathcal{Y}=\frac{u d^{2}(d-1)^{2}}{(u+v-u v)^{2}},
$$

we obtain the equation

$$
d(d-1) u v=(u v-u-v)(1+d(u v-u-v))
$$


or

$$
(u v-u-v)(d v-1)(d u-1)=d(d-1) u v(u-1)(v-1) .
$$

In [8], we give fibrations with elliptic parameter in the multiplicative group generated by $\Pi=\{u, v, u-1, v-1, u d-1, v d-1, d, d-1\}$. Let us recall the divisors of functions of $\prod$,

$$
\begin{gathered}
\operatorname{div}(u)= \\
(0)+(5 A)-2(6 A)-\Theta_{1,6}-\Theta_{0,4}-2 \Theta_{0,5}-\Theta_{0,6} \\
+2 \Theta_{\infty, 0}+\Theta_{\infty, 1}-\Theta_{\infty, 3}+\Theta_{\infty, 5}+2 \Theta_{\infty, 6} \\
\operatorname{div}(v)= \\
(0)-2(A)+(2 A)-\Theta_{1,1}-\Theta_{0,1}-2 \Theta_{0,2}-\Theta_{0,3} \\
+2 \Theta_{\infty, 0}+2 \Theta_{\infty, 1}+\Theta_{\infty, 2}-\Theta_{\infty, 4}+\Theta_{\infty, 6} \\
\operatorname{div}(u-1)= \\
(A)+(4 A)-2(6 A)+\Theta_{1,1}+\Theta_{1,2}+\Theta_{1,3}+\Theta_{1,4}-\Theta_{1,6} \\
+\Theta_{0,1}+\Theta_{0,2}-\Theta_{0,4}-2 \Theta_{0,5}-\Theta_{0,6}-\Theta_{\infty, 3} \\
\operatorname{div}(v-1)= \\
(3 A)+(6 A)-2(A)-\Theta_{1,1}+\Theta_{1,3}+\Theta_{1,4}+\Theta_{1,5}+\Theta_{1,6} \\
+\Theta_{0,5}+\Theta_{0,6}-\Theta_{0,1}-2 \Theta_{0,2}-\Theta_{0,3}-\Theta_{\infty, 4} \\
\operatorname{div}(d u-1)= \\
(2 A)+(3 A)-2(6 A)+\Theta_{1,1}+2 \Theta_{1,2}+2 \Theta_{1,3}+\Theta_{1,4}-\Theta_{1,6} \\
-\Theta_{0,5}-\Theta_{\infty, 2}-2 \Theta_{\infty, 3}-\Theta_{\infty, 4} \\
\operatorname{div}(d v-1)= \\
(4 A)+(5 A)-2(A)+\Theta_{1,3}+2 \Theta_{1,4}+2 \Theta_{1,5}+\Theta_{1,6}-\Theta_{1,1} \\
-\Theta_{0,2}-\Theta_{\infty, 3}-2 \Theta_{\infty, 4}-\Theta_{\infty, 5}
\end{gathered}
$$

We show in [8] that $k=(d-1) /(u-1)$ is an elliptic parameter for an elliptic fibration with reducible fibers of types $I_{10}, I_{2}^{*}$ and $I_{3}$ at, respectively, $k=\infty, 0,-1$ represented in Figure 1 . The reducible fiber of type $I_{3}$ has three components: $\Theta_{1,2}, \Theta_{1,3}$ and $\Gamma$. More precisely, from $k=-1$, we have the parametrization of $\Gamma$

$$
u=\frac{v(2 v-1)}{v^{2}-v+1}, v, d=\frac{v-2}{v^{2}-v+1} .
$$


So we can say the divisor of $k+1$ is equal to:

$$
\begin{aligned}
- & (A)-(4 A)-\Theta_{0,1}-\Theta_{0,2}-\Theta_{\infty, 0}-\Theta_{\infty, 1}-\Theta_{\infty, 2}-\Theta_{\infty, 4}-\Theta_{\infty, 5}-\Theta_{\infty, 6} \\
& +\Theta_{1,2}+\Theta_{1,3}+\Gamma
\end{aligned}
$$

and also compute the divisor of $d+u-2=(k+1)(u-1)$.

We add the function $k+1$ to the set of functions $\prod$ and compute new fibrations as in [8] using Proposition 2, with parameters in the multiplicative group generated by $\prod \cup\{k+1\}$. In this section, horizontal and vertical divisors always refer to the fibration of parameter $k$.

We start from the fibration of parameter $k$ with the three Weierstrass equations

$$
\begin{aligned}
Y^{2}+(k+1)(k-2)\left(X+k^{2}\right) Y & =\left(X+k^{2}\right)\left(X+k^{2}-1\right)\left(X+k^{2}+k^{3}\right), \\
Y^{2}+(k+1)(k-2) Y x & =x(x-1)\left(x+k^{3}\right), \\
y^{2}+k(k+1) y x & =x(x+k)\left(x-k^{2}\right)
\end{aligned}
$$

where $x=X+k^{2}$ and $y=Y-(k+1) x$.

For this calculation, we choose

$$
\begin{aligned}
& X=(k+1) \frac{(v-1) d}{u-1}, \quad X+k^{2}=\frac{v(1+d(u v-u-v))}{u(u-1)}, \\
& X+k^{2}+k=(k+1) \frac{v d-1}{u-1}, \\
& Y+\left(k^{2}-1\right) x=(k+1) \frac{d(v d-1) v}{u(u-1)} .
\end{aligned}
$$

The zero section $O_{k}$ corresponds to the component $\Theta_{1,1}$, the section $(2 A)$ gives a two-torsion section $T=\left(X=-k^{2}, Y=0\right)$, the section (5A) corresponds to $P_{k}=\left(X=Y=-k-k^{2}\right)$.

We draw graphs (Figure 1) of reducible fibers of type $I_{10}, I_{2}^{*}$ and $I_{3}$ at $k=\infty, 0,-1$; we also draw on the graphs some sections $\left(0_{k}, T, P_{k}\right)$ from the fibration of parameter $k$. 


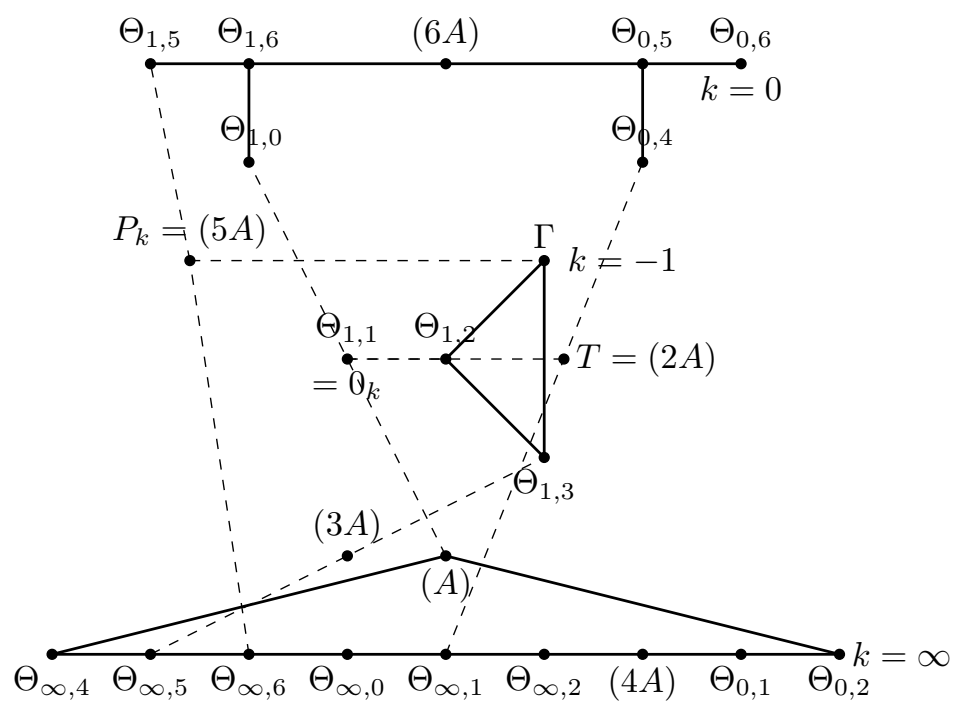

FiguRe 1. Fibration of parameter $k$.

\subsection{A first set.}

9.2.1. Fibration of parameter $w$. Let $w^{-}$and $w^{+}$be the two divisors of types $I_{13}$ and $I_{6}$

$$
\begin{aligned}
w^{-}= & (A)+\Theta_{1,1}+\Theta_{1,0}+\Theta_{1,6}+(6 A)+\Theta_{0,5} \\
& +\Theta_{0,4}+(2 A)+\Theta_{\infty, 1}+\Theta_{\infty, 2}+(4 A)+\Theta_{0,1}+\Theta_{0,2} \\
w^{+}= & (5 A)+\Theta_{\infty, 6}+\Theta_{\infty, 5}+(3 A)+\Theta_{1,3}+\Gamma .
\end{aligned}
$$

The horizontal divisor of $w^{+}-w^{-}$is $(5 A)+(3 A)-(2 A)-\Theta_{1,1}$ $=P_{k}+(3 A)-T-O_{k}$ and corresponds to the horizontal divisor of the function

$$
w_{0}=\frac{Y+\left(k^{2}-1\right) x}{x} .
$$

Considering the previous list of divisors and the divisor of $k+1$, we can choose $w=\left(w_{0}\right) / k$. Using the transformation $Z=\left(x-k^{2}\right) / x$, we get an equation of bidegree 2 in $Z$ and $k$. After transformation, we obtain 
the following Weierstrass equation

$$
y^{\prime 2}+\left(w^{2}-w+1\right) y^{\prime} x^{\prime}-w y^{\prime}=x^{\prime 2}\left(x^{\prime}-w\right) .
$$

9.2.2. Fibration of parameter $a$. We consider the two divisors of types $I_{11}$ and $I I I^{*}$

$$
\begin{aligned}
a^{+}= & \Gamma+\Theta_{1,3}+(3 A)+\Theta_{0,6}+\Theta_{0,5}+\Theta_{0,4}+(2 A)+\Theta_{\infty, 1}+\Theta_{\infty, 0} \\
& +\Theta_{\infty, 6}+(5 A) \\
a^{-}= & (4 A)+2 \Theta_{0,1}+3 \Theta_{0,2}+4(A)+3 \Theta_{1,1}+2 \Theta_{1,0}+\Theta_{1,6}+2 \Theta_{\infty, 4}
\end{aligned}
$$

The horizontal divisor of $a^{+}$is $\delta_{1}=(3 A)+T+P_{k}$, the horizontal divisor of $a^{-}$is $\delta_{2}=3 \Theta_{1,1}$ and $\delta_{1}-\delta_{2}$ is the horizontal divisor of the function $a_{0}$ equal to $Y-(k+1) x=y$. From the equation in $k, x, y$, we can calculate the divisor of $a_{0}$, then, choosing $a=\left(a_{0} / k^{2}\right)$, we obtain a function with divisor $a^{+}-a^{-}$and

$$
a=\frac{(d u-1)(d v-1)(d-2+u)}{(u-1)^{2}(d-1)^{2}} .
$$

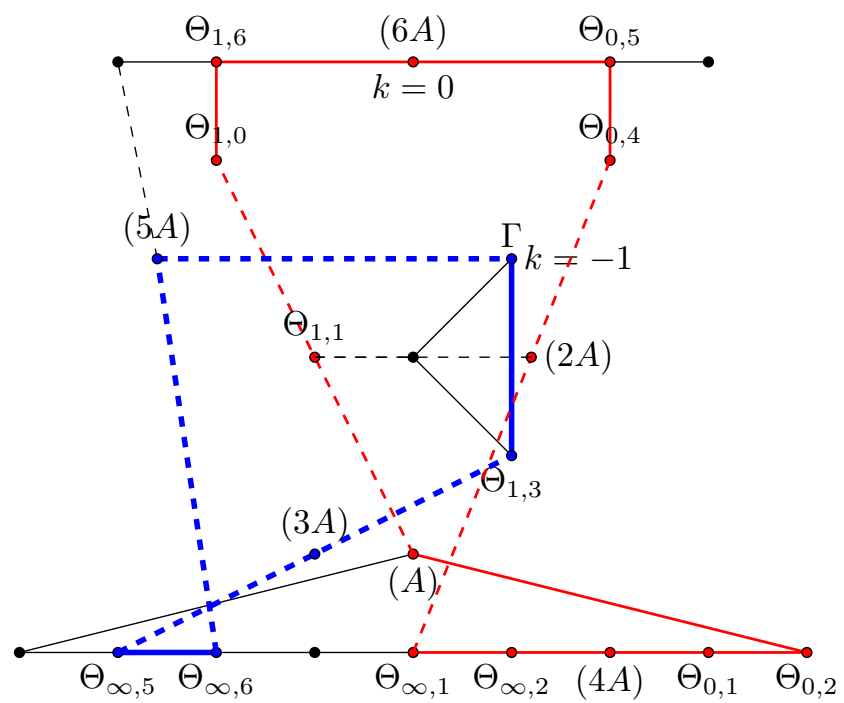

FiguRE 2. Fibration of parameter $w$. 


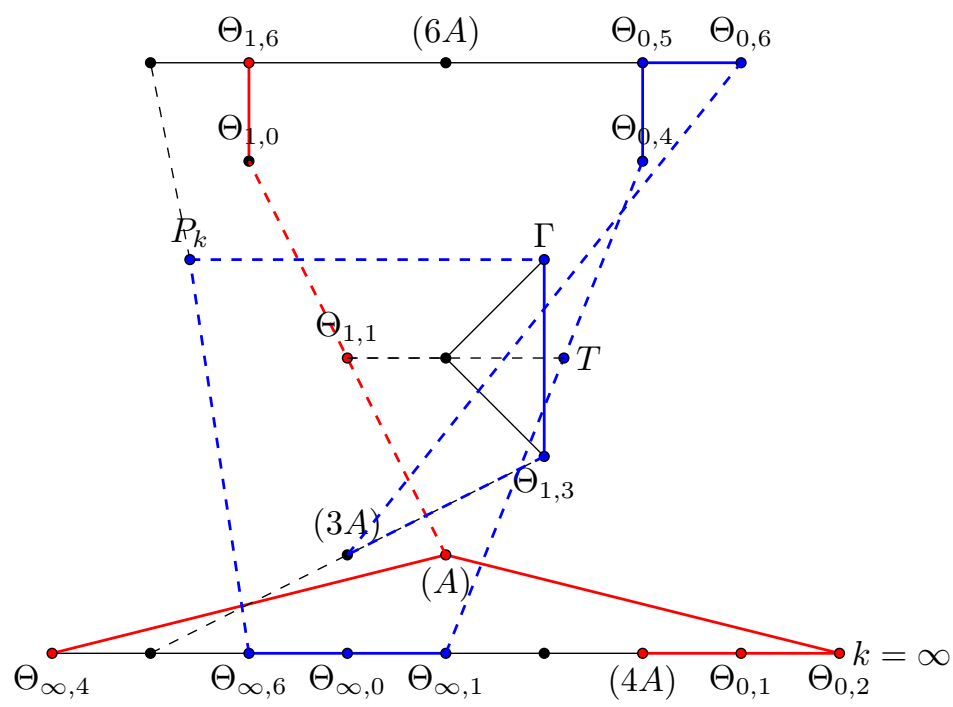

FIGURE 3. Fibration of parameter $a$.

Then, we have the equation

$$
a_{0}^{2}+k(k+1) a_{0} x=x(x+k)\left(x-k^{2}\right) .
$$

Setting $k^{2} z=x$ and $k=1 /(z Q)$ follows an equation of bidegree 2 in $z$ and $Q$. After classical transformations

$$
X^{\prime}=\frac{(x+k)}{k^{2}}, \quad Y^{\prime}=\frac{(x+k) a}{k x},
$$

then, we obtain

$$
Y^{\prime 2}+\frac{a+1}{a} Y^{\prime} X^{\prime}+Y^{\prime}=X^{\prime}\left(X^{\prime 2}-X^{\prime}-a\right)
$$

or also, with $y^{\prime}=\left(Y^{\prime} / a^{3}\right), x^{\prime}=\left(X^{\prime} / a^{2}\right)$,

$$
y^{\prime 2}+(a+1) y^{\prime} x^{\prime}+a^{3} y^{\prime}=x\left(x^{2}-a^{2} x-a^{5}\right) .
$$

Moreover, we can compute the divisors of $X^{\prime}$ and $Y^{\prime}$ since we have the 
formulas

$$
\begin{aligned}
X^{\prime} & =\frac{(d-2+u)(d v-1)}{(d-1)^{2}} \\
Y^{\prime} & =\frac{(d-2+u)^{2}(d v-1) u(v-1)}{(d-1)^{3} v(u-1)} .
\end{aligned}
$$

So we can deduce that the 0 section $\left(=0_{a}\right)$ of the fibration of parameter $a$ is $(6 A)$ and that $\Theta_{1,4}$ corresponds to $P_{a}=\left(x^{\prime}=0, y^{\prime}=0\right)$ and $-P_{a}=\left(0,-a^{3}\right)$.

\subsection{An elliptic divisor and a part of another elliptic divisor.} In the following example, to determine the elliptic parameter, we use the fact that an elliptic curve has no function with a single simple pole.

9.3.1. Fibration of parameter $b$. Let $b^{-}$be the effective divisor

$$
\begin{aligned}
\Theta_{0,1}+(4 A)+\Theta_{\infty, 2}+\Theta_{\infty, 1}+\Theta_{\infty, 0}+\Theta_{\infty, 6}+\Theta_{\infty, 5}+\Theta_{\infty, 4} \\
+(A)+\Theta_{1,1}+\Theta_{1,0}+\Theta_{1,6}+(6 A)+\Theta_{0,5}+\Theta_{0,6}+\Theta_{0,0}
\end{aligned}
$$

which represents a singular fiber of type $I_{16}$ of a fibration of parameter $b$. The horizontal divisor of $b^{-}$is $\Theta_{1,1}+\Theta_{0,0}=0_{k}+\left(P_{k}+T\right)$. We see on the graph that $\Theta_{0,3} \cdot b^{-}=0$, so the component $\Theta_{0,3}=\left(-2 P_{k}+T\right)$ is part of another singular fiber of this new fibration. There is a unique function (up to constant in $\mathbb{C}(k)$ ) on $E(\mathbb{C}(k)$ ) with divisor $-0_{k}-\left(P_{k}+T\right)+\left(-2 P_{k}+T\right)+\left(3 P_{k}\right)$. This function $b_{0}$ is equal to $\left(Y+(k-1) X+k^{4}-k^{2}\right) / X$, and so $b$ is equal to $b_{0} k^{r}$. At $k=\infty$, we have a singular fiber of type $I_{10}$. Let $K=1 / k, Y=\left(Y_{1} / K^{6}\right)$ and $X=\left(X_{1} / K^{4}\right)$. Then $b_{0}=\left(Y_{1}-X_{1}\left(K-K^{2}\right)+K^{2}-K^{4}\right) /\left(K^{2} X_{1}\right)$. We must have a simple pole at $\Theta_{\infty, 4}$; the component is obtained after blowing $X_{1}=K X_{2}, Y_{1}=K Y_{2}$, so $b=b_{0} K, r=-1$ and

$$
b=\frac{Y+(k-1) X+k^{4}-k^{2}}{X k} .
$$

We eliminate $Y$ between the Weierstrass equation in $Y, X$ and the definition of $b$. Setting $X=k^{2} U$, we get an equation in $k$ and $U$ of bidegree 2. After transformation, we obtain a Weierstrass form

$$
Y^{2}-b(b-3) Y X=X(X-1)^{2} .
$$


We see that there is a singular fiber at $b=0$ of type $I_{2}$ with components the two sections $\left(-2 P_{k}+T\right)$ and $\left(3 P_{k}\right)$, since the two sections $\left(-2 P_{k}+\right.$ $T)$ and $\left(3 P_{k}\right)$ are intersecting in $k=1 / 2$.

We also find

$$
b=\frac{(d u-1)(d v-1)(u v-u-v+d)(-u-v+2 u v)}{(u-1)^{2} d(v-1)^{2}(d-1) u v}
$$

9.3.2. Fibration of parameter $p$. Let us define the divisor $p^{-}$corresponding to a singular fiber of type $I_{5}^{*}$

$$
\begin{aligned}
p^{-}= & \Theta_{1,5}+\Theta_{1,0}+2 \Theta_{1,6}+2(6 A)+2 \Theta_{0,5}+2 \Theta_{0,6}+2(3 A)+2 \Theta_{\infty, 5} \\
& +\Theta_{\infty, 4}+\Theta_{\infty, 6} .
\end{aligned}
$$

The divisor

$$
R_{2}=\Theta_{1,2}+(2 A)+\Theta_{\infty, 1}+\Theta_{\infty, 2}+(4 A)+\Theta_{0,1}+\Theta_{0,2}+\Theta_{0,3}
$$

is orthogonal to $p^{-}$and is a part of another singular fiber.

The horizontal divisor of $-p^{-}+R_{2}$ is $-2(3 A)+(2 A)+\Theta_{0,3}$ and thus is equal to $-2\left(-P_{k}+T\right)+T+\left(-2 P_{k}+T\right)$. It is the horizontal divisor of the function

$$
p_{0}=\frac{\left(-k^{2} Y+\left(X+k^{2}+k^{3}\right)\left(k^{2}+X\right)\right)}{X^{2}} .
$$

We can choose $p=p_{0} k^{r}(k+1)^{s}$. As for $b$ at $k=\infty$, we get $r=-1$, and $p$ must have a zero on $\Theta_{1,2}$ so $s=1$. Therefore,

$$
p=\frac{(k+1)\left(-k^{2} Y+\left(X+k^{2}+k^{3}\right)\left(k^{2}+X\right)\right)}{k X^{2}}
$$

is a parameter of a new fibration with a singular fiber of type $I_{5}^{*}$ and another of type $I_{9}$.

We obtain the following Weierstrass equation

$$
y_{1}^{2}+\left(1+\frac{1}{p}\right) y_{1} x_{1}-\frac{1}{p}=x_{1}^{2}\left(x_{1}+(p-1)\right) .
$$

Note that

$$
p=\frac{(d v-1)(d u-1)^{2}(-u-v+2 u v)}{(u-1)^{2}(d-1) d^{2}(v-1)^{2} u v}
$$


9.4. Fibrations in families of $K 3$ surfaces. Our $K 3$ surface is also obtained by specializing the parameter of two families of $K 3$ surfaces. Each of these families has an elliptic fibration valid for all members of the family (see also $[\mathbf{2}, \mathbf{3}, \mathbf{7}]$ ). Let $K$ be a parameter.

We start with the first family

$$
y^{2}=x^{3}+\left(t+\frac{1}{t}+K\right) x^{2}+x
$$

with singular fibers in general of type $2 I_{4}^{*}, 4 I_{1}$, a two-torsion point and two points of abscissae $\left(x=-t^{3}, x=-t\right)$. We can verify that $n=x / t$ is an elliptic parameter of a new fibration with singular fibers $2 I I I^{*}, I_{4}$, a two-torsion section and equation

$$
Y^{2}=X^{3}+K X^{2}+\frac{(n+1)^{2}}{n} X .
$$

From [8], the case we are interested in is $K=9 / 4$ (see also Table 3 ).

From $S_{K}$, we can recover the fibration of parameter $b$ with $b^{\prime}=y / x$ and $b=b^{\prime}-3 / 2$. The two singular fibers of type $I_{4}^{*}$, the 0 -section and the two-torsion section give in the new fibration a singular fiber of type $I_{16}$. A Weierstrass equation for this fibration is

$$
y^{\prime 2}+\left(b^{\prime 2}-K\right) y^{\prime} x^{\prime}=x^{\prime}\left(x^{\prime}-1\right)^{2} .
$$

If $K$ is a square, $K=j^{2}$, we can recover a fibration with one fiber of type $I V^{*}$ and one of type $I_{12}$. The Weierstrass equation of the fibration of parameter $n$ can be transformed in

$$
y^{\prime \prime 2}-2 j n y^{\prime \prime} x^{\prime \prime}=x^{\prime \prime 3}-(n+1)^{2} n^{3} x^{\prime \prime},
$$

and then with a new parameter equal to $y^{\prime \prime} /\left(x^{\prime \prime}(n+1)\right)$ we can recover for $K=9 / 4$ the fibration of parameter $m$ of [8].

9.4.1. Fibration of parameter $q$. From the fibration of parameter $n$ and the Weierstrass equation

$$
Y^{2}=X^{3}+X^{2} K+\frac{(n+1)^{2}}{n} X,
$$

define $X=q$ as a new parameter. This corresponds to an elliptic fibration with two singular fibers of type $I_{8}^{*}, I_{1}^{*}$ and equation

$$
y^{2}=x^{3}+q\left(K q+2+q^{2}\right) x^{2}+q^{2} x .
$$


For $K=9 / 4$, we obtain the result for our modular surface.

9.4.2. Fibration of parameter $l$. From the fibration of parameter $n$ and Weierstrass equation

$$
Y^{2}=X^{3}+X^{2} K+\frac{(n+1)^{2}}{n} X,
$$

let $l=X / n$ be a new parameter. Then we have an elliptic fibration with two singular fibers of types $I_{5}^{*}$ and $I I^{*}$ of equation

$$
y^{2}=x^{3}+\left(\frac{1}{l}+K\right) x^{2}+2 x+l .
$$

For $K=9 / 4$, we obtain the result for our modular surface.

9.4.3. Fibration of parameter $o$. Let $K^{\prime}$ be a parameter, we look at the second family of elliptic $K 3$ surfaces

$$
Y^{2}=X^{3}+K^{\prime} X^{2}+\frac{h^{2}-2 h+1}{h} .
$$

Our $K 3$ surface corresponds to $K^{\prime}=-15 / 4$ (cf., Table 3 ). This elliptic surface has two singular fibers of type $I I^{*}$, at $h=0, h=\infty$ and a singular fiber of type $I_{2}$. Taking $X$ to be a new parameter, we get an elliptic fibration with two singular fibers of types $I_{12}^{*}, I_{2}$ and equation

$$
y^{2}=x^{3}+\left(o^{3}+K^{\prime} o^{2}-2\right) x^{2}+x .
$$

For $K^{\prime}=-(15) / 4$, we have a new fibration of our modular surface.

9.4.4. Fibration of parameter $f$. Starting with the fibration of parameter $o$

$$
y^{2}=x^{3}+\left(o^{3}+K^{\prime} o^{2}-2\right) x^{2}+x,
$$

we take $f=y / x o$ as a new parameter. Using the transformations

$$
x=Y^{\prime}, \quad o=\frac{1-Y^{\prime}}{X^{\prime}},
$$

we obtain a new fibration with Weierstrass equation

$$
Y^{\prime 2}+\left(-K^{\prime}+f^{2}\right) X^{\prime} Y^{\prime}-Y^{\prime}=X^{3} .
$$


For $K^{\prime}=-15 / 4$, we have a new fibration of our modular surface with one singular fiber of type $I_{18}$ and six singular fibers of type $I_{1}$.

9.5. The two last fibrations. From now on, the terms 'horizontal' and 'vertical' refer to the fibration of parameter $a$.

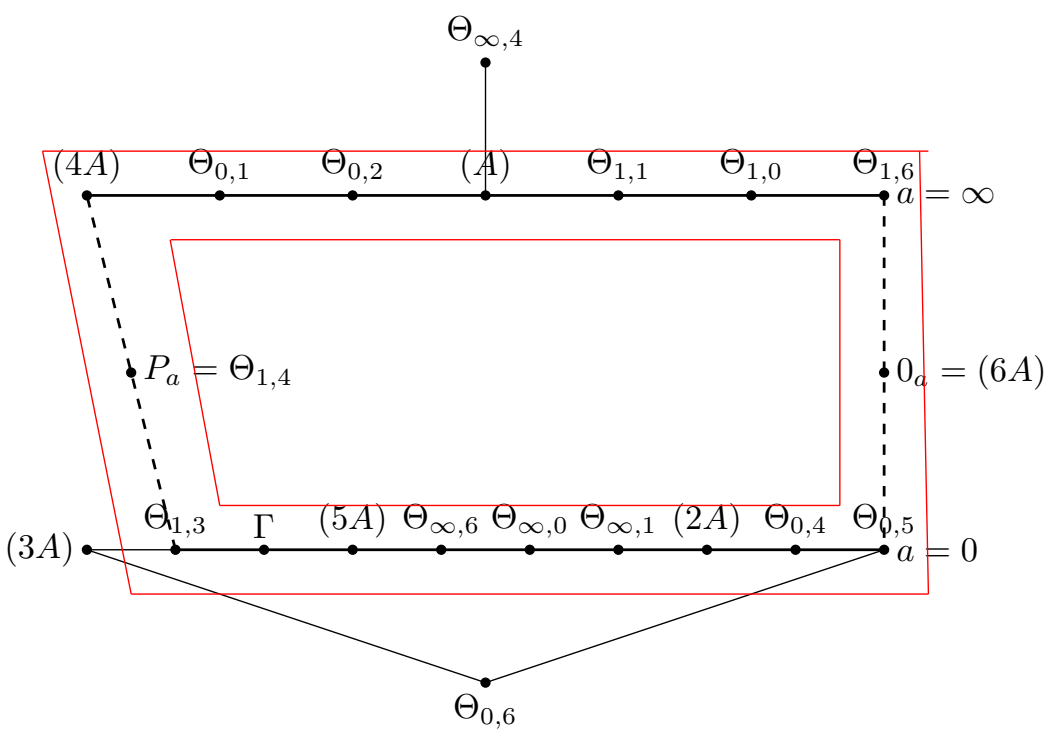

FigURE 4. From fibration of parameter $a$ to fibration of parameter $g$.

9.5.1. Fibration of parameter $g$. Let

$$
\begin{aligned}
g^{-}= & (4 A)+\Theta_{0,1}+\Theta_{0,2}+(A)+\Theta_{1,1}+\Theta_{1,0}+\Theta_{1,6}+(6 A) \\
& +\Theta_{0,5}+\Theta_{0,4}+(2 A)+\Theta_{\infty, 1}+\Theta_{\infty, 0}+\Theta_{\infty, 6}+(5 A) \\
& +\Gamma+\Theta_{1,3}+\Theta_{1,4} .
\end{aligned}
$$

This divisor corresponds to a singular fiber of type $I_{18}$. The horizontal divisor of $g^{-}$is equal to $(6 A)+\Theta_{1,4}=0_{a}+P_{a}$. No component of the Coxeter graph is in the orthogonal of $g^{-}$. From previous computations and equation (9.6) we see we can choose as parameter for this new fibration $g=\left(y^{\prime}+a^{3}\right) /\left(a x^{\prime}\right)+s a+r / a$, with $(r, s) \in \mathbb{C}^{2}$. First we look at the singular fiber of type $I_{11}$ at $a=0$ to determine $r$ 
Using the three blow ups $x^{\prime}=a X_{1}, y^{\prime}=a Y_{1} ; x^{\prime}=a^{2} X_{2}$, $y^{\prime}=a^{2} Y_{2}$ and $x^{\prime}=a^{3}\left(-1+X_{3}\right), y^{\prime}=a^{3} Y_{3}$ in the equation (9.6), we determine that the section $-P_{a}$ intersects $\Theta_{\infty, 1}$. So, the component $\Theta_{\infty, 1}$ corresponds to $Y_{3}+X_{3} \equiv 0 \bmod a$.

The component $\Theta_{0,6}$ corresponds to $x^{\prime}=a X_{1}$ and $y^{\prime}=a Y_{1}$ with $Y_{1} \equiv 0 \bmod a$, so $\left(y^{\prime}+a^{3}\right) /\left(x^{\prime} a\right)$ has no pole on this component and then $r=0$.

We look now at the singular fiber of type $I I I^{*}$ at $a=\infty$ to determine $s$. Let $a=1 / \widetilde{a}, x^{\prime}=x / \widetilde{a}^{4}, y^{\prime}=y / \widetilde{a}^{6}$. The equation becomes

$$
y^{2}+\widetilde{a}(\widetilde{a}+1) y x+\widetilde{a}^{3} y=x\left(x^{2}-\widetilde{a}^{2} x-\widetilde{a}^{3}\right) .
$$

The function $\left(y^{\prime}+a^{3}\right) /\left(a x^{\prime}\right)+s a$ is then equal to $\left(y+\widetilde{a}^{3}\right) /(\widetilde{a} x)+s / \widetilde{a}$. The components $\Theta_{1,6}$ and $(4 A)$ obtained after blowing up $x=\widetilde{a} x_{1}, y=$ $\widetilde{a} y_{1}$ correspond to $Y_{1}^{2} Z_{1} \equiv 0 \bmod \widetilde{a}$ with $x_{1}=\left(X_{1} / Z_{1}\right), y_{1}=\left(Y_{1} / Z_{1}\right)$ and are simple poles of the function $\left(y+\widetilde{a}^{3}\right) /(\widetilde{a} x)$.

We need to choose $s$ such that the component $\Theta_{\infty, 4}$ is not a pole for $y+\widetilde{a}^{3} \widetilde{a} x+s / \widetilde{a}$. An easy way is to calculate divisors of $y /(\widetilde{a} x)$ and $\widetilde{a}^{3} /(\widetilde{a} x)$ from $X^{\prime}$ and $Y^{\prime}$ using $u, v(9.7)$ and (9.5). We can see that $\Theta_{\infty, 4}$ is not a pole for $y /(\widetilde{a} x)$ and neither for $\widetilde{a}^{3} /(\widetilde{a} x)$, so $s=0$ and

$$
g=\frac{y+\widetilde{a}^{3}}{\widetilde{a} x}=\frac{y^{\prime}+a^{3}}{a x^{\prime}} .
$$

If $Y^{\prime \prime}=X^{\prime} a$ and $X^{\prime \prime}=a$, we have the Weierstrass equation for this new fibration

$$
Y^{\prime \prime 2}-\left(g^{2}+g+1\right) X^{\prime \prime} Y^{\prime \prime}-g Y^{\prime \prime}=X^{\prime \prime 3}-(g+1) X^{\prime \prime 2}+X^{\prime \prime}
$$

9.5.2. Fibration of parameter $e$. In Figure 5, we surround a singular fiber of type $I_{13}^{*}$ corresponding to a divisor $e^{-}$.

$$
\begin{aligned}
& e^{-}=(3 A)+\Theta_{1,4}+ \\
& 2\left(\Theta_{1,3}+\Gamma+(5 A)+\Theta_{\infty, 6}+\Theta_{\infty, 0}+\Theta_{\infty, 1}+(2 A)\right. \\
& \left.\quad+\Theta_{0,4}+\Theta_{0,5}+(6 A)+\Theta_{1,6}+\Theta_{1,0}+\Theta_{1,1}+(A)\right) \\
& \quad+\Theta_{0,2}+\Theta_{\infty, 4} .
\end{aligned}
$$


We see the horizontal divisor of $e^{-}$is $P_{a}+20_{a}$, so we can choose a parameter $e$ of the type $\left(y^{\prime}+a^{3}\right) /\left(a^{2} x^{\prime}\right)+s\left(x / a^{2}\right)+m a^{2}+t a+r / a^{2}+$ $w / a$.

As seen above, the two functions $\left(y^{\prime}+a^{3}\right) /\left(a^{2} x^{\prime}\right)$ and $x / a^{2}$ have simple poles on $\Theta_{0,6}$, so $r=0$. On this component $y^{\prime}=a^{2} X_{1}^{2} \bmod a^{2}$ and $x^{\prime}=a X_{1}$, so $w=0$ and $s=-1$. Using again the previous results for $\Theta_{\infty, 4}$ obtained for $g$, we see that $x / a^{2}$ has a simple pole at $\Theta_{\infty, 4}$ and then $m=0$.

Using (9.7) and the transformation $(u, v) \mapsto(\mathcal{X}, \mathcal{Y})$, we can prove the section $(4 A)$ is not a pole for $\left[\left(y^{\prime}+a^{3}\right) /\left(a^{2} x^{\prime}\right)\right]-x / a^{2}$, so $t=0$ and

$$
e=\frac{y^{\prime}+a^{3}}{a^{2} x^{\prime}}-\frac{x}{a^{2}}
$$

is a parameter for the last fibration. Notice that we also have $e=$ $\left(Y^{\prime \prime}-g\right) / X^{\prime \prime}$. Letting $X^{\prime \prime}=z+(g / e)$, we have an equation of bidegree

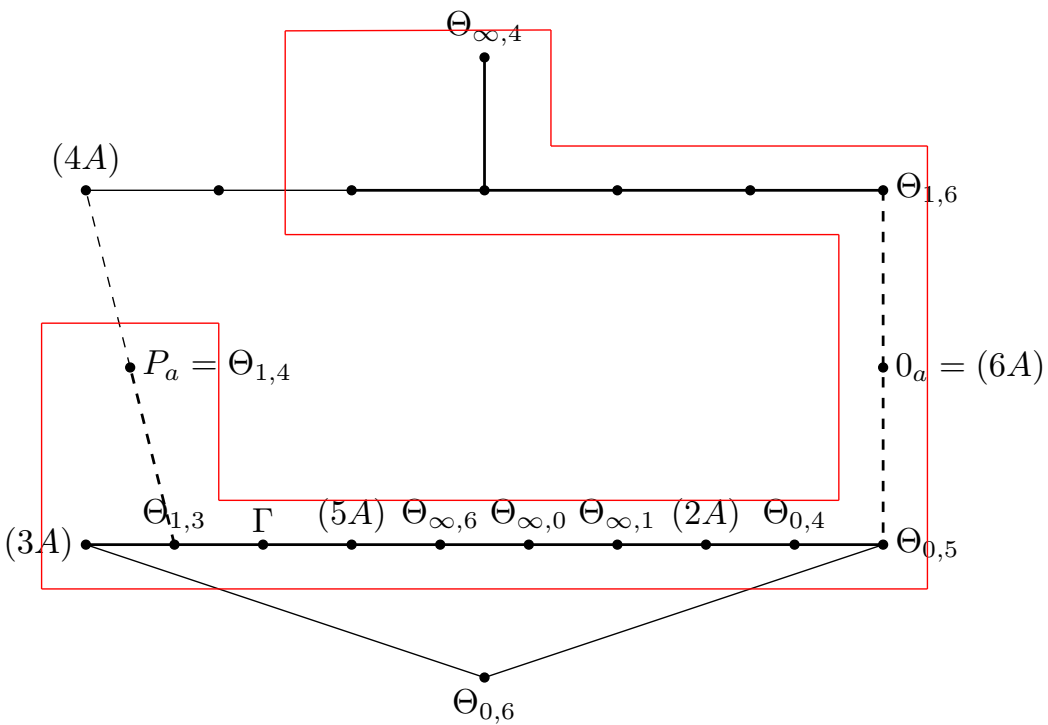

FiguRE 5. From fibration of parameter $a$ to fibration of parameter $e$. 
2 in $g, z$. With usual transformation we obtain a Weierstrass equation (9.11)

$Y^{2}+\left(\frac{(e+2)(e-1) X}{e}+1\right) Y=\left(X+\frac{e-1}{e^{2}}\right)\left(X^{2}-e\left(e^{2}+e+1\right) X-e^{2}\right)$.

TABLE 3. Previous results from [8].

\begin{tabular}{|c|c|c|c|}
\hline Weierstrass Equation & Singular fibers & $\begin{array}{l}\text { Torsion } \\
\text { Rank }\end{array}$ & $\begin{array}{l}\text { tor. generator } x \text {-coord } \\
\text { inf. generator } x \text {-coord }\end{array}$ \\
\hline $\begin{array}{l}y^{2}+\left(1+d-d^{2}\right) y x \\
\quad+\left(d^{2}-d^{3}\right) y \\
=x^{3}+\left(d^{2}-d^{3}\right) x^{2}\end{array}$ & $3 I_{7}, 3 I_{1}$ & $\begin{array}{c}\mathbb{Z} / 7 \mathbb{Z} \\
0\end{array}$ & $\begin{array}{c}0 \\
-\end{array}$ \\
\hline $\begin{array}{l}y^{2}+v y x-(v-1)^{2} v^{2} y \\
=x^{3}-(v-1)^{2} v x^{2}\end{array}$ & $2 I_{1}^{*}, I_{8}, 2 I_{1}$ & $\begin{array}{c}\mathbb{Z} / 4 \mathbb{Z} \\
1\end{array}$ & $\begin{array}{c}0 \\
-v(v-1)\end{array}$ \\
\hline $\begin{array}{l}y^{2}+k(k+1) y x \\
=x(x+k)\left(x-k^{2}\right)\end{array}$ & $I_{10}, I_{2}^{*}, I_{3}, 3 I_{1}$ & $\begin{array}{c}\mathbb{Z} / 2 \mathbb{Z} \\
1\end{array}$ & $\begin{array}{c}0 \\
-k\end{array}$ \\
\hline $\begin{array}{c}y^{2}-m(m-3) y x \\
+m^{2} y=x^{3}\end{array}$ & $I_{12}, I V^{*}, 4 I_{1}$ & $\begin{array}{c}\mathbb{Z} / 3 \mathbb{Z} \\
1\end{array}$ & $\begin{array}{c}0 \\
-m+1\end{array}$ \\
\hline $\begin{array}{l}y^{2}+s y x \\
\quad+s^{3}(1-s) y \\
=x^{3}+s(1-s) x^{2}\end{array}$ & $I V^{*}, I_{5}, I_{3}^{*}, 2 I_{1}$ & $\begin{array}{l}0 \\
1\end{array}$ & - \\
\hline $\begin{array}{l}y^{2}+\left(2 t^{2}+t+2\right) y x \\
=x(x-1)\left(x-t^{4}\right)\end{array}$ & $2 I_{4}^{*}, 4 I_{1}$ & $\begin{array}{c}\mathbb{Z} / 2 \mathbb{Z} \\
2\end{array}$ & $\begin{array}{c}0 \\
1,-t^{3}\end{array}$ \\
\hline $\begin{array}{c}y^{2}-\left(r^{2}-r+1\right) y x \\
+r^{3} y=x^{3}\end{array}$ & $2 I_{9}, I_{2}, 4 I_{1}$ & $\begin{array}{c}\mathbb{Z} / 3 \mathbb{Z} \\
1\end{array}$ & $\begin{array}{l}0 \\
r\end{array}$ \\
\hline $\begin{array}{l}y^{2}=x^{3}+\frac{9}{4} n^{2} x^{2} \\
\quad+(n+1)^{2} n^{3} x\end{array}$ & $2 I I I^{*}, I_{4}, 2 I_{1}$ & $\begin{array}{c}\mathbb{Z} / 2 \mathbb{Z} \\
1\end{array}$ & $\begin{array}{c}0 \\
n^{3}(n+1)\end{array}$ \\
\hline $\begin{array}{l}y^{2}=x^{3}-\frac{15}{4} x^{2} h^{2} \\
\quad+(h-1)^{2} h^{5}\end{array}$ & $2 I I^{*}, I_{2}, 2 I_{1}$ & $\begin{array}{l}0 \\
1\end{array}$ & $\frac{1}{9}(h-1)^{2}\left(h^{2}-7 h+1\right)$ \\
\hline $\begin{aligned} y^{2} & =x^{3}+c\left(-\frac{15}{4} c+1\right) x^{2} \\
& -c^{5} x\end{aligned}$ & $I I I^{*}, I_{6}^{*}, 3 I_{1}$ & $\begin{array}{c}\mathbb{Z} / 2 \mathbb{Z} \\
1\end{array}$ & $\begin{array}{c}0 \\
-\frac{c(2 c-1)^{2}}{(c+1)^{2}}\end{array}$ \\
\hline
\end{tabular}

All the results are summarized in Tables 3 and 4. For each fibration, $x$-coordinate of torsion points and generators of Mordell-Weil group are given in the last column. Most of the points are obtained from transformations giving the Weierstrass equations; the others follow from a direct calculation. Using results of [18] or [16, Section 11], we calculate the heights of these points and deduce they are generators since the discriminant of $N S(S)$ is 7 . 
TABLE 4. Other fibrations.

\begin{tabular}{|c|c|c|c|}
\hline Weierstrass Equation & Singular fibers & $\begin{array}{l}\text { Torsion } \\
\text { Rank }\end{array}$ & $\begin{array}{l}\text { tor. generator } x \text {-coord } \\
\text { inf. generator } x \text {-coord }\end{array}$ \\
\hline $\begin{array}{l}y^{2}+\left(w^{2}-w+1\right) y x-w y \\
=x^{3}-w x^{2}\end{array}$ & $I_{13}, I_{6}, 5 I_{1}$ & $\begin{array}{l}0 \\
1\end{array}$ & $\overline{0}$ \\
\hline $\begin{array}{l}y^{2}+(a+1) y x+a^{3} y \\
=x\left(x^{2}-a^{2} x-a^{5}\right)\end{array}$ & $I_{11}, I I I^{*}, 4 I_{1}$ & $\begin{array}{l}0 \\
1\end{array}$ & $\begin{array}{l}- \\
0\end{array}$ \\
\hline $\begin{array}{l}y^{2}-b(b-3) y x \\
=x(x-1)^{2}\end{array}$ & $I_{16}, 2 I_{2}, 4 I_{1}$ & $\begin{array}{c}1 \\
\mathbb{Z} / 4 \mathbb{Z}\end{array}$ & $\begin{array}{c}1 \\
(b-1)^{2}\end{array}$ \\
\hline $\begin{array}{l}y^{2}+(p+1) y x-p^{2} y \\
=x^{3}+p^{2}(p-1) x^{2}\end{array}$ & $I_{5}^{*}, I_{9}, 4 I_{1}$ & $\begin{array}{l}0 \\
1\end{array}$ & $\overline{0}$ \\
\hline $\begin{array}{l}y^{2}=x^{3}+q\left(q^{2}+\frac{9}{4} q+2\right) x^{2} \\
\quad+q^{2} x\end{array}$ & $I_{8}^{*}, I_{1}^{*}, 3 I_{1}$ & 1 & $-q(q+1)^{2}$ \\
\hline $\begin{array}{l}y^{2}=x^{3}+l\left(1+\frac{9}{4} l\right) x^{2} \\
\quad+2 l^{4} x+l^{7}\end{array}$ & $I I^{*}, I_{5}^{*}, 3 I_{1}$ & $\begin{array}{l}0 \\
1\end{array}$ & $\begin{array}{c}- \\
l^{3}(l-1)\end{array}$ \\
\hline $\begin{aligned} y^{2} & =x^{3}+\left(o^{3}-\frac{15}{4} o^{2}-2\right) x^{2} \\
& +x\end{aligned}$ & $I_{12}^{*}, I_{2}, 4 I_{1}$ & $\begin{array}{c}\mathbb{Z} / 2 \mathbb{Z} \\
1\end{array}$ & $\begin{array}{c}0 \\
\frac{1}{9}(o-1)^{2}(o-3)^{2}\end{array}$ \\
\hline$y^{2}+\left(\frac{15}{4}+f^{2}\right) y x-y=x^{3}$ & $I_{18}, 6 I_{1}$ & $\begin{array}{c}\mathbb{Z} / 3 \mathbb{Z} \\
1\end{array}$ & $\begin{array}{c}0 \\
4 \frac{\left(4 f^{2}-9\right)}{\left(4 f^{2}+3\right)^{2}}\end{array}$ \\
\hline $\begin{array}{l}y^{2}+\left(g^{2}+g+1\right) y x+g y \\
=x\left(x^{2}-(g+1) x-1\right)\end{array}$ & $I_{18}, 6 I_{1}$ & $\begin{array}{l}0 \\
1\end{array}$ & - \\
\hline $\begin{aligned} y^{2}= & x^{3}-\frac{1}{4}(e+1) \\
& \left(4 e^{2}-e+3\right) x^{2} \\
& +\frac{1}{2}\left(-4 e^{2}+e+1\right) x+\frac{5}{4}-e\end{aligned}$ & $I_{13}^{*}, 5 I_{1}$ & 0 & $\begin{array}{c}- \\
-\frac{e-1}{e^{2}}\end{array}$ \\
\hline
\end{tabular}

Acknowledgments. The author would like to express her gratitude to Professor Marie José Bertin for her many helpful comments. The computer algebra system Maple and Maple Library "Elliptic Surface Calculator," written by Masato Kuwata [10] were used in the calculations for this paper.

\section{REFERENCES}

1. M.J. Bertin and O. Lecacheux, Elliptic fibration of the modular surface associated to $\Gamma_{1}(8)$, in Arithmetic and geometry of K3 surfaces and Calabi-Yau threefolds, 153-199, Fields Inst. Comm. 67, Springer, New York, 2013.

2. A. Clingher and C. Doran Modular invariants for lattice polarized K3 surfaces, Mich. Math. J. 55 (2007), 355-393.

3. , Note on a geometric isogeny of K3 surfaces, Int. Math. Res. Not. 16 (2011), 3657-3687. 
4. J.H. Conway and N.J.A. Sloane, Sphere packings, lattices and groups, Grund. Math. Wissen. 290, Springer-Verlag, Berlin, 1993.

5. N.D. Elkies, Mordell-Weil generators for singular Shioda-Inose surfaces over $\mathbb{Q}$, http://www.math.harvard.edu/ ${ }^{\sim}$ elkies/K3_20SI.html.

6. A. Garbagnati and A. Sarti, Elliptic fibrations and symplectic automorphisms on K3 surfaces, Comm. Alg. 37 (2009), 3601-3631.

7. T. Harrache, Etude des fibrations d'une surface $K 3$, Thèse de l'Université Paris 6, 27 Nov. 2009.

8. T. Harrache and O. Lecacheux, Etude des fibrations elliptiques d'une surface K3, J. Th. Nomb. Bord. 23 (2011), 183-207.

9. A. Kumar, Elliptic fibration on a generic jacobian Kummer surface, preprint, arXiv: 1105.1715 .

10. M. Kuwata, Maple library, 'Elliptic surface calculator,' http://cfaculty. chuo-u.ac.jp/ kuwata/ESC.php.

11. J. Martinet, Les réseaux parfaits des espaces euclidiens, Masson, Paris, 1996.

12. H.-V. Niemeier, Definite quadratische Formen der Dimension 24 und Diskriminante 1, J. Num. Th. 5 (1973), 142-178.

13. V.V. Nikulin, Integral symmetric bilinear forms and some of their applications, Math. USSR Izv. 14 (1980), 103-167.

14. K.-I. Nishiyama, The Jacobian fibrations on some K3 surfaces and their Mordell-Weil groups, Japan. J. Math. 22 (1996), 293-347.

15. I.-I. Piatetski-Shapiro and I.-R. Shafarevich, Torelli's theorem for algebraic surfaces of type K3, Izv. Akad. Nauk SSSR 35 (1971), 530-572.

16. M. Schütt and T. Shioda, Elliptic surfaces, arXiv: $0907.0298 v 3$ [math. AG], 9 Jul. 2010.

17. T. Sengupta, Elliptic fibrations on supersingular $K 3$ surface with Artin invariant 1 in characteristic 3, preprint, arXiv:1204.6478.

18. T. Shioda, On the Mordell-Weil lattices, Comm. Math. Univ. St. Paul. 39 (1990), 211-240.

Université Pierre et Marie Curie (Paris 6), Institut de Mathématiques, 4 Place Jussieu, 75005 Paris, France

Email address: odile.lecacheux@imj-prg.fr 